\title{
MECC scheduling algorithm in vehicular environment for uplink transmission in LTE networks
}

\author{
Shafinaz Ismail, Darmawaty Mohd Ali, Azita Laily Yusof \\ Wireless Communication Technology Group, Universiti Teknologi MARA, Malaysia
}

\begin{tabular}{l}
\hline \hline Article Info \\
\hline Article history: \\
Received May 24, 2018 \\
Revised Oct 9, 2018 \\
Accepted Oct 31, 2018 \\
\hline
\end{tabular}

\section{Keywords:}

Contiguity constraint LTE

Scheduling algorithm Uplink transmission Vehicular environment

\begin{abstract}
Single Carrier Frequency Division Multiple Access (SC-FDMA) is chosen because of the lower peak-to-average power ratio (PAPR) value in uplink transmission. However, the contiguity constraint is one of the major constraint presents in uplink packet scheduling, where all RBs allocated to a single UE must be contiguous in the frequency-domain within each time slot to maintain its single carrier. This paper proposed an uplink-scheduling algorithm namely the Maximum Expansion with Contiguity Constraints (MECC) algorithm, which supports both the RT and NRT services. The MECC algorithm is deployed in two stages. In the first stage, the RBs are allocated fairly among the UEs. The second stage allocates the RBs with the highest metric value and expands the allocation on both sides of the matrix, $\mathrm{M}$ with respect to the contiguity constraint. The performance of the MECC algorithm was observed in terms of throughput, fairness, delay, and Packet Loss Ratio (PLR) for VoIP, video and best effort flows. The MECC scheduling algorithm is compared to other algorithms namely the Round Robin (RR), Channel-Dependent First Maximum Expansion (CD-FME), and Proportional Fairness First Maximum Expansion (PF-FME). From here, it can be concluded that the MECC algorithm shows the best results among other algorithms by delivering the highest throughput which is up to $81.29 \%$ and $90.04 \%$ than CD-FME and RR scheduler for RT and NRT traffic respectively, having low PLR and delay which is up to $93.92 \%$ and $56.22 \%$ of improvement than CD-FME for the RT traffic flow. The MECC also has a satisfactory level of fairness for the cell-edge users in a vehicular environment of LTE network.
\end{abstract}

Copyright $(5) 2019$ Institute of Advanced Engineering and Science. All rights reserved.

\section{Corresponding Author:}

Shafinaz Ismail,

Faculty of Electrical Engineering, Universiti Teknologi MARA,

40450 Shah Alam, Selangor, Malaysia.

Email: shafinaz.ismail@gmail.com

\section{INTRODUCTION}

Long Term Evolution (LTE) is the most capable wireless broadband, which is developed by the Third Generation Partnership Project (3GPP) [1]. In LTE technology, Single Carrier Frequency Division Multiple Access (SC-FDMA) offers lower peak-to-average power ratio (PAPR) as compared to Orthogonal Frequency Division Multiple Access (OFDMA) thus, making SC-FDMA is more suitable for the uplink transmission. The SC-FDMA system delivers higher throughput, lower PAPR, higher spectral efficiency, and lower bit error rate than the conventional OFDMA technique [2]. In spite of the advantages of SC-FDMA, it requires that all subcarriers allocated to a single UE must be adjacent to each other in the frequency domain [3].

The system architecture of the 3GPP LTE system contains several base stations called the LTE base station (eNodeB) where the packet scheduling is performed with other Radio Resource Management (RRM) 
tasks. LTE network supports different types of services that share the radio access and core network resources. These services are the real time (RT) and non-real time (NRT) where each services have different quality of service (QoS) requirements in terms of bit rate, delay, and PLR, which must be provisioned [4]. The network architecture for LTE consists of Evolved Node B (eNodeB), Evolved Packet System (EPS) and the UEs. The LTE transmission is divided into frames, which consist of 10 subframes. A subframe duration is $1 \mathrm{~ms}$ in length. Each subframe is divided into two slots where each slot is $0.5 \mathrm{~ms}$ in length. A subframe is also known as the Transmission Time Interval (TTI). The physical layer interface is a transport block with common Modulation and Coding Scheme (MCS). Each TTI contains at most one transport block per UE [5]. Each slot present in frequency domain is divided into a number of resource blocks. The structure of a time slot in frequency domain is divided into regions of $180 \mathrm{kHz}$ that contain a contiguous set of 12 subcarriers. Hence the total number of uplink physical resource blocks (PRBs) ranges between 6 PRBs for the smallest (1.4 MHz) and $100 \mathrm{PRBs}$ for the largest bandwidth $(20 \mathrm{MHz})$ respectively.

In OFDM-based multi-user framework, schedulers play an important role in optimizing the network performance and provide Quality of Service (QoS) requirements in the Medium Access Control (MAC) layer. The MAC scheduler in the eNodeB is deployed in the uplink and downlink transmission is mainly responsible for allocating resource block (RBs) among UEs to support the diverse QoS requirements. The task of the scheduler depends on the specific algorithm used and the Channel Quality Indicator (CQI), which provides feedback from UEs on whether the channel condition is good or poor, and allocate RBs accordingly [6], [7]. The contiguity constraint is one of the major constraints in the uplink scheduling. This constraint refers to having all PRBs, allocated to a single UE to be adjacent along the frequency domain. The contiguity constraint can reduce the spectral efficiency of the uplink transmission, since UE is being allocated a PRB despite the existence of other UEs with better channel quality over the same PRB [8].

The uplink packet scheduling is still facing some challenges that can limit its performance due to the single carrier property of SC-FDMA. Therefore, the scheduler has to consider the needs of the users to satisfy their QoS requirements while maintaining the efficient and fair allocation of PRBs. Several papers have been discussed in literature. Three channel aware namely the Recursive Maximum Expansion (RME), First Maximum Expansion (FME) and Minimum Area Difference (MAD) is proposed by L.De Temiño et al. in [9]. The results showed that the performance of RME, FME, and MAD are improved in terms of spectral efficiency and fairness. A modified version of FME namely the Modified FME (MFME) was proposed by K. Elgazzar et al. in [10]. MFME modified the expansion step of the FME, which continues to search between the non-idle UEs to allocate the RBs. The proposed scheme has better performance than the FME in terms of throughput, fairness and spectral efficiency. H. Safa and K. Tohme [11] have presented a performance evaluation of FME, RME and Riding Peak with both Channel-Dependent (CD) and Proportional Fairness (PF) paradigm matrix. The FME algorithm has the worst performance as compared to other algorithms in term of throughput, fairness, delay, and mobility in both paradigms Matrix. R. Ahmad et al. [12] evaluated the RR, MT and FME scheduling performance, which focused on the throughput and fairness for different service classes. The FME and MT deliver the lowest throughput for RT services and the lowest fairness for all traffic flows. S. Dardouri et al. [13] have evaluated the performance of several downlink scheduling algorithms in LTE network for VoIP, video and best effort applications in terms of throughput, fairness, delay and packet loss rate (PLR) in pedestrian and vehicular environment. T. Janevski et al. [14] have presented a performance evaluation of several downlink scheduling algorithms for High Speed Downlink Packet Access (HSDPA) network in pedestrian and vehicular environment. S. Mahesha et al. [15] have studied the impact of handover on performance of downlink scheduling algorithms in pedestrian and vehicular environment.

Nevertheless, none of the aforementioned studies have taken into consideration the throughput, delay, packet loss ratio (PLR) and fairness index of the cell-edge users in vehicular environment in the uplink transmission. In this paper, a new scheduling algorithm, namely the Maximum Expansion Contiguity Constraint (MECC) is proposed to satisfy the contiguity constraint and improve the throughput, fairness index, delay, and packet loss ratio of the cell-edge users in the uplink transmission. The rest of the paper is organized as follows. Section 2 explains the proposed algorithm. The simulation results are described in Section 3 and finally, the paper concludes in Section 4.

\section{RESEARCH METHOD}

In this paper, the Blind Equal Throughput (BET) algorithm is used as the scheduling metric. The BET algorithm aims to provide equal throughput to all UEs in the system. A scheduler receives an input matrix $\mathrm{M}$ with a dimension of $\left[\mathrm{N}_{\mathrm{UE}} \mathrm{x} \mathrm{N}_{\mathrm{RB}}\right]$, where the input, $M_{i, k}$ is calculated using the BET equation, as shown in Figure 1.

Int J Elec \& Comp Eng, Vol. 9, No. 2, April 2019 : 1191 - 1200 


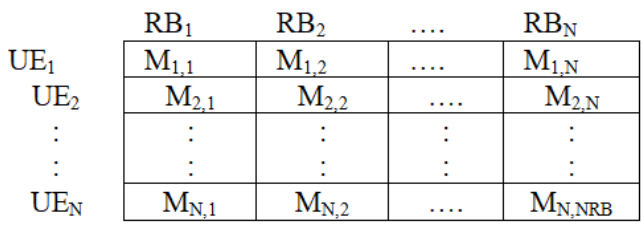

Figure 1. Input matrix of $\mathrm{M}$

\subsection{Blind equal throughput (BET) algorithm}

The BET scheduler aims to provide an equal throughput to all UEs in the system. The priority metric for BET is given by

$$
m_{i, k}^{B E T}=\frac{1}{R_{i}(t)}
$$

where $R_{i}(t)$ is the past average throughput of UE $i$ at time $t$ which is calculated by

$$
R_{i}(t)=\beta R_{i}(t-1)+(1-\beta) r^{i}(t)
$$

Hence, $B(0<\beta<1)$ is the weight factor for moving average, and $r^{i}(t)$ is the achievable data rate of UE $i$ at time $t$ as defined above. The value of $\beta$ is set to 0.1 in order to achieve the best performance of throughput and fairness [16]. In every transmission time interval (TTI), BET scheduler allocates the resources to flows that have been served with lower average throughput in the past. UE experiencing the lowest throughput will be served as long as the user does not reach the same throughput of other users in the cell. In this way, users with bad channel conditions are allocated more often.

\subsection{Uplink resource allocation in LTE systems}

The MECC scheduler has two stage of operation. In the first stage, the available RBs are divided into groups of RBs according to $\mid \frac{\left|N_{R B}\right|}{\left|N_{U E}\right|}$, where $N_{R B}$ is the number of RB and $N_{U E}$ is the number of UEs. Then, it distributes the groups of RBs among the available UEs. The fair distribution of RBs among UEs is to ensure that the same amounts of RBs are given to all users. In the second stage of allocation, the multi-user diversity gain is exploited by allocating the RBs among several UEs depending on the channel conditions. The RBs are assigned starting from the highest metric value and expanding the allocation on both sides of matrix M. Each UE is considered served whenever another UE having better metric value is found. In this stage, the allocation begins by searching the combination of UE-RB with the highest metric value from $M_{i, k}$ as shown in Figure 1 and expanding the allocation on both the right and the left sides from the input of matrix $\mathrm{M}$ and assigning the corresponding channel the RB $\left(N_{R B}\right)$ and $\mathrm{UE}(N)$. As the scheduler traverses through each RB, it checks its maximum value in the first column on the right-hand side and on the left-hand side of matrix M. If the maximum value on the right-hand side is higher than the maximum value on the left-hand side, it will expand on the right-hand side of matrix $\mathrm{M}$, otherwise on the left-hand side. The scheduler checks each column of matrix $\mathrm{M}$ and determines whether the maximum metric still belongs to the UE in which resources are currently being assigned, or whether the maximum metric belongs to another UE. If the conditions are fulfilled, the RB is assigned to the selected UE; otherwise, the UE is considered served, and the current RB is assigned to a new UE. The scheduler then reiterates the expansion procedure. Assigning the RB to the other UE would break the continuity constraints. The flow chart of the MECC algorithm is depicted in Figure 2. 


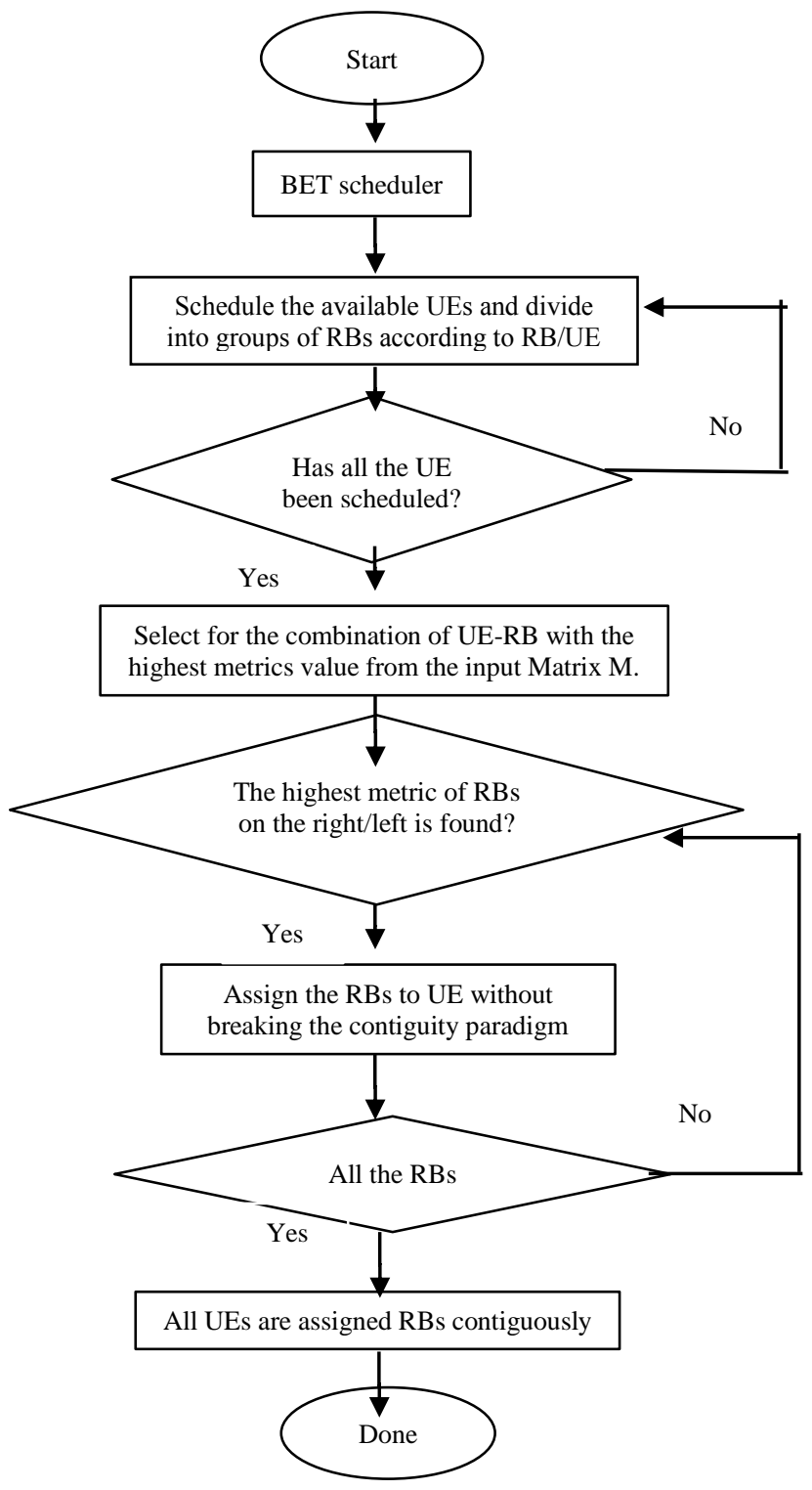

Figure 2. The flow chart of the MECC algorithm

\subsection{Simulation parameters}

In this paper, video and VoIP flows are used for the RT services while infinite-buffer, which is known as the Best Effort (BE) flow is representing the NRT. The trace-based application delivers packets based on a video trace file, which is obtained from [5]. The voice flows of G.729 are generating VoIP application. An ON/OFF Markov chain is modeled for the voice flow, where the ON period has a mean value of $3 \mathrm{~s}$ and is exponentially distributed. The OFF period has a truncated exponential probability distribution function with an average value of $3 \mathrm{~s}$ and an upper limit of $6.9 \mathrm{~s}$ [17]. The source delivers 20 bytes sized packet every $20 \mathrm{~ms}$ during the $\mathrm{ON}$ period, which is equivalent to $8 \mathrm{kbps}$, while the data rate is zero during the OFF period because of the Voice Activity Detector. Finally, the Infinite-Buffer application model demonstrates a greedy source that constantly possesses packets to be delivered [5]. The performance of MECC, RR, PF-FME, and CD-FME are evaluated based on throughput, fairness index, Packet Loss Ratio (PLR) and delay [18]. The performance metric of throughput represents the rate of the successful packet being delivered over a physical channel. The parameter is calculated by dividing the number of successfully received bits with the duration of the flow and can be mathematically expressed as:

$$
\text { Throughput }=\frac{P_{\text {transmit }}}{t}
$$


where $P_{\text {transmit }}$ is the size of the transmitted packets and $t$ is the time it takes to transfer the packets per each user.

The fairness index indicates that every user in the system receives a fair share of the resources. Fairness index is obtained by considering the throughput achieved by each flow at the end of each simulation. Fairness among users is implemented using the Jain's Fairness Index and it is expressed as:

$$
\text { Fairness Index }=\frac{\left(\sum x_{i}\right)^{2}}{n x \sum x_{i}^{2}}
$$

where, $x_{i}$ is the throughput of the user, $i$, and $n$ is the number of active flows.

The delay is the amount of time a packet takes to traverse a system. If packets are not transmitted within the deadline, the packets will be discarded. The Packet Loss Ratio (PLR) measures the percentage of packets of data traveling across a physical channel, which could not reach their destination. This is calculated as given:

$$
P L R=\left(\frac{P_{\text {transmit }}-P_{\text {receive }}}{P_{\text {transmit }}}\right) \times 100
$$

where $P_{\text {receive }}$ is the size of the received packets.

A single cell of $1 \mathrm{~km}$ of radius with eNodeB located at the center of the cell is modeled in the simulation. The number of UE is varied from 20 to 200. The movement of UE in the cell is adopting the random direction model. The speed is set to $120 \mathrm{~km} / \mathrm{h}$, which resembles the vehicular scenarios. The bandwidth is set to $10 \mathrm{MHz}$. User Datagram Protocol (UDP) is used as a transport protocol [19]. Each user receives one H.264 video flow encoded at $242 \mathrm{kbps}$, one G.729 voice flows and one BE flow. The simulations considered four different phenomena including the path loss in urban area, penetration loss, shadowing fading and the effect of fast fading due to the signal multipath. The fast fading channel, which is Jakes model, is used as the propagation model. The simulation parameters used in LTE-SIM are summarized in Table 1.

Table 1. LTE Uplink Simulation Parameters

\begin{tabular}{cc}
\hline Parameter & Value \\
\hline Simulation Duration & 80 seconds \\
Transmission Power & $43 \mathrm{dBm}$ \\
Frequency Carrier & $1.98 \mathrm{GHz}$ \\
Number of RBs & 50 \\
RB Bandwidth & $180 \mathrm{KHz}$ \\
Time Transmission Interval & $1 \mathrm{~ms}$ \\
Maximum Delay & $0.1 \mathrm{~s}$ \\
\hline
\end{tabular}

\section{RESULTS AND ANALYSIS}

In this section, the performance of the MECC is compared to the RR, PF-FME, and CD-FME in terms of throughput as demonstrated in Figure 3 to Figure 11 respectively.

\subsection{Throughput}

Figure 3, Figure 4, and Figure 5 show the throughput of VoIP, video and BE flows respectively. The throughput of VoIP flows is illustrated in Figure 3. It is observed that the throughput of VoIP for MECC and RR increases exponentially as the number of user increases and the performances are comparable to each other up to 120 users. However, after that, the throughput of RR is $4.23 \%$ higher than MECC because there is no packet being dropped as shown in Figure 9, hence more packets can be sent out. Nonetheless, the performance of PF-FME and CD-FME are $97.56 \%$ and $98.02 \%$ lower than MECC.

In the case of video flows, as shown in Figure 4, the throughput of MECC outperforms other scheduling algorithms. However, the performance of MECC has decreases slightly as the number of the user increases due to the interference from the network signal with the increasing speed of UEs. The throughput of MECC shows significant improvement, which is $81.29 \%, 76.96 \%$, and $90.04 \%$ higher as compared to the CD-FME, PF-FME and RR schedulers respectively. The throughput of video for RR gradually decreases and has the lowest throughput after 140 users, which is $56.75 \%$ lower as compared to the PF-FME because of packet dropped as shown in Figure 10. This resulted in less utilization of assigned PRBs. The CD-FME and PF-FME show the same trending, which in turn, assigned unallocated resources to the BE flows. 
Figure 5 illustrates the throughput for BE flows. The throughput of the MECC is the highest as compared to the RR, PF-FME, and CD-FME, which are $96.69 \%, 49.96 \%$ and $43.41 \%$ lower than the MECC respectively. The throughput of RR is the lowest among the others. It is noticed that the throughput of RR starts to decrease as the number of user increases due to the non-stringent delay requirement of the NRT traffic. As a result, the RR algorithm provides a high quota to the VoIP and video flows. On the other hand, both CD-FME and PF-FME deliver the lowest throughput for RT since both algorithms prioritized the NRT flows and leaving few RBs to VoIP and video flows.

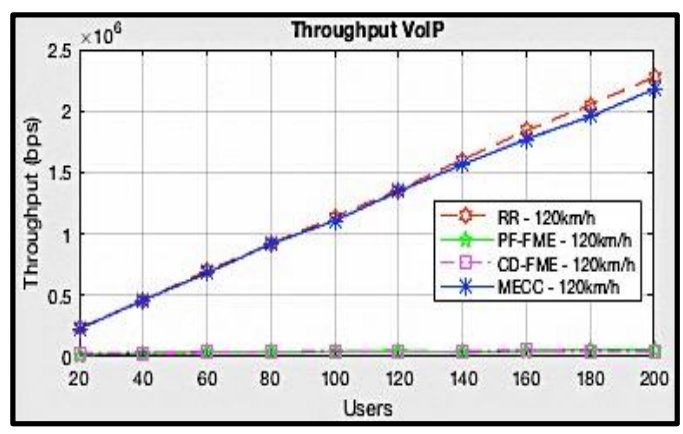

Figure 3. Throughput for VoIP flows

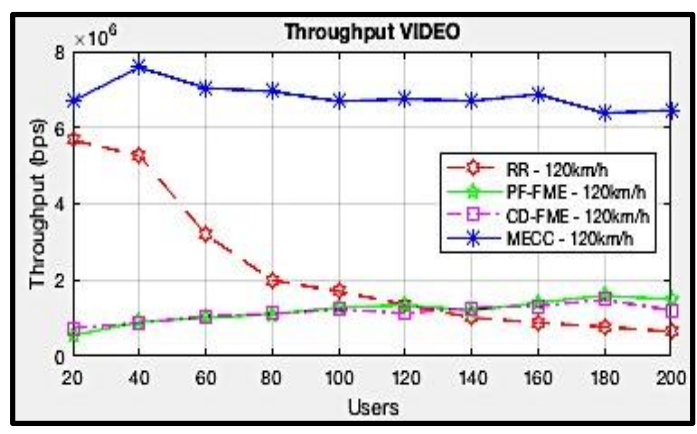

Figure 4. Throughput for video flows

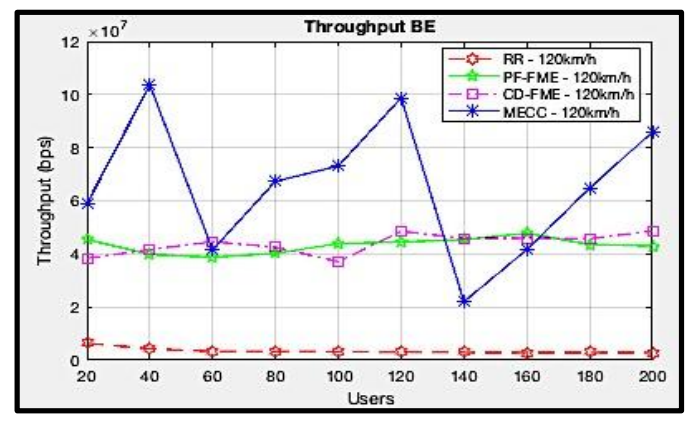

Figure 5. Throughput for BE flows

\subsection{Delay}

RT service is an application that has a strict delay requirement, which needs to be provisioned. Figure 6, Figure 7 and Figure 8 show the delay of VoIP, video and BE flows respectively. The delay for VoIP flows is illustrated in Figure 6. It is noticed that VoIP users suffer longer latency as more users joined the network. The delay of VoIP for all scheduling algorithms gradually increases when the network is loaded with 200 users. The CD-FME and PF-FME have the highest delay, whereas RR delivers the lowest. The delay of MECC increases gradually and it is 50.08\% and 56.22\% lower than PF-FME and CD-FME respectively. The delay of VoIP flows must be given the highest priority because the packet associated with the voice traffic is obviously smaller than the delay of the video flows. Therefore, it must be assigned to a guaranteed bandwidth channel in order to make sure that the packet delivery is within a tolerable delay limit.

In the case of video flows as demonstrated in Figure 7, it is noticed that video flows have a longer latency when there are more users in the cell. The RR scheduler has the highest packet delay, which is 61.14\% higher than MECC while CD-FME and PF-FME deliver the lowest, which are $26.47 \%$ and $54.67 \%$ lower than MECC. The delay of the RR is higher than MECC because RR prioritizes the non-GBR traffic instead of GBR traffic. On the other hand, the MECC algorithm shows a constant delay of $0.03 \mathrm{~s}$ between 60 to 200 users. The MECC algorithm does not deliver the lowest delay because when throughput is improved, data packets are scheduled in order to ensure throughput is maximized and this affects the delay performance. There is a tradeoff between throughput and delay, where the delay will increase when the throughput is maximized. The QoS value for the VoIP and video delay is set to be less than $100 \mathrm{~ms}$ and $150 \mathrm{~ms}$ respectively as specified by the 3GPP standard (20). Therefore, all algorithms are observed to have a low delay, which is in the acceptable range to provision the Quality of User Experience (QoE). 
The delay of BE flows is presented in Figure 8. The delay of BE flows is modeled using the infinite buffer model, thus the delay will always be a constant value of $1 \mathrm{~ms}$ for all scheduling strategies [21].

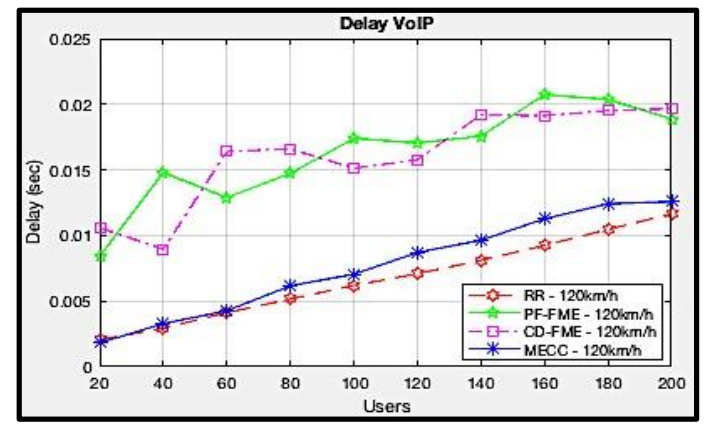

Figure 6. Delay for VoIP flows

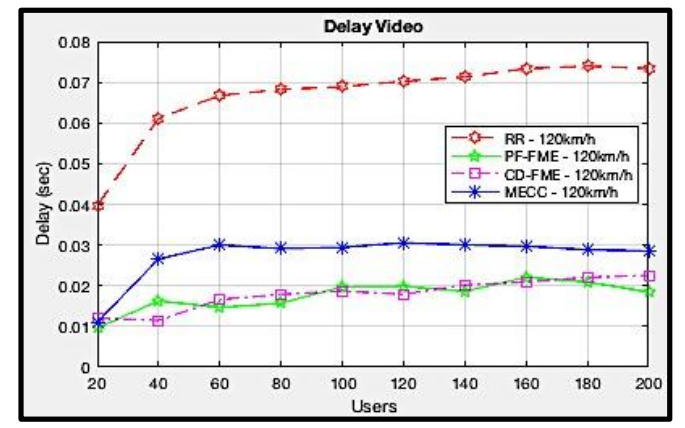

Figure 7. Delay for video flows

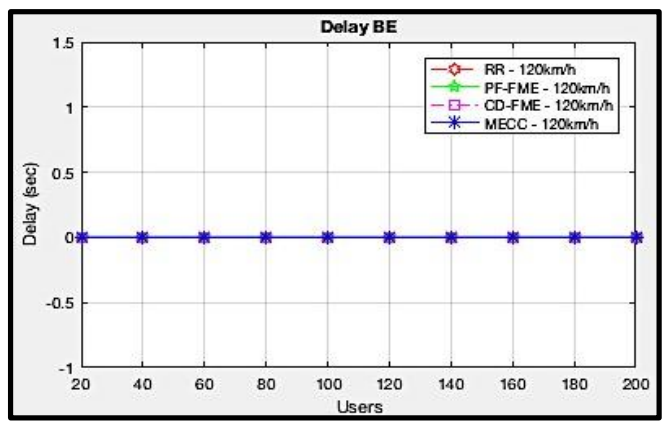

Figure 8. Delay for BE flows

\subsection{Packet loss ratio}

PLR is an important parameter for the RT flows because it is an indicator whether the packets are delivered timely. The PLR of VoIP, video and BE flows are depicted in Figure 9, Figure 10 and Figure 11 respectively. The PLR of VoIP flows is presented in Figure 9. It is observed that both CD-FME and PF-FME achieved the highest PLR that is up to $93.92 \%$ than MECC and this is in accordance with the throughput performanceThe higher throughput being sent out will result in lower PLR. The PLR of MECC scheduler is approaching zero when the number of user is less than 80 , however, the PLR starts to violate the allowable value when the number of UE exceeds 100 since the acceptable value should be less than $1 \times 10^{-2 .}$ The MECC scheduler managed to keep the PLR value less than $0.55 \%$ at 80 user and increases after 80 users, which is up to $5.97 \%$ than the RR algorithm. The RR has the lowest PLR, which is 5.97\% lower than MECC and is kept constant at zero.

For the video application, all the PLR increases as the number of user increases due to the highspeed movements of the vehicles. As a result, when the speed increases, frequent changes of dynamic topology causes frequent interruptions of the communication. Thus, when the speed increases, the possibility to discard packet for deadline expiration increases as demonstrated in Figure 10. MECC scheduler has the lowest PLR, which is $6.55 \%, 9.73 \%$ and $10.04 \%$ lower than RR, CD-FME, and PF-FME respectively. Both CD-FME and PF-FME has the highest PLR, which contribute to low throughput. The VoIP flows experienced significantly lower PLR than video flows because the VoIP traffic is delivering lower source bit rate as, compared to video flows as illustrated in Figure 9 and Figure 10. The PLR for video flows has violated the QoS value as specified by the 3GPP. The acceptable range of VoIP and video flows should not exceed $1 \times 10^{-2}$ to $1 \times 10^{-3}$ respectively [20].

The PLR of BE flow is illustrated in Figure 11. It can be observed that RR scheduler has the highest PLR, followed by the MECC and CD-FME and PF-FME respectively. The PLR of RR increases gradually and is $85.83 \%$ higher than the MECC because RR algorithm delivers the lowest throughput. The PLR of MECC is $95.42 \%$ higher than CD-FME, however, the MECC managed to keep the PLR value to less than $0.5 \%$ when the network is loaded with 200 users. Both CD-FME and PF-FME schedulers have the lowest 
PLR and this is in accordance with the throughput performance. Moreover, there is no QoS requirement need to be provisioned by the BE flows.

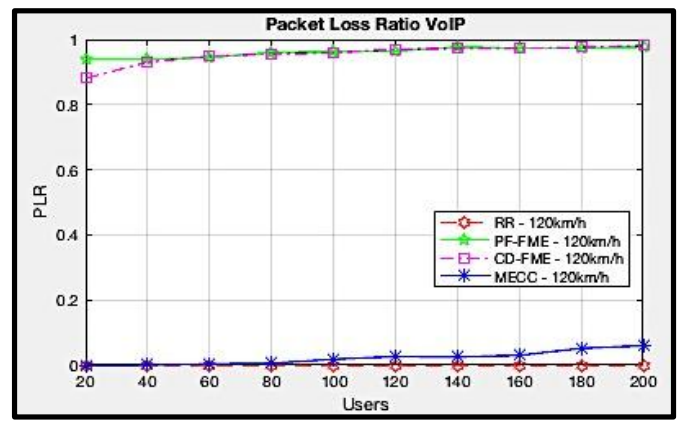

Figure 9. PLR for VoIP flows

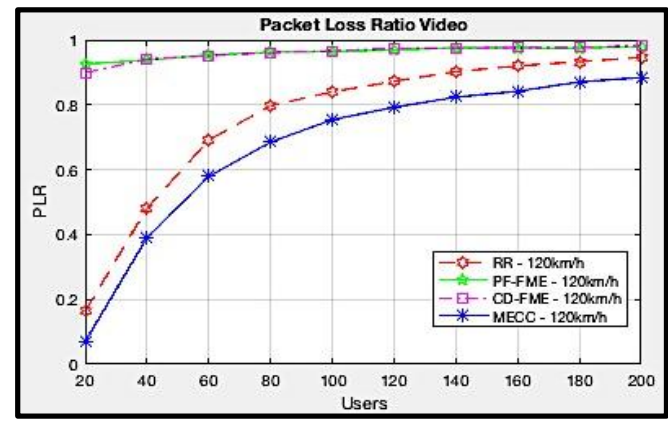

Figure 10. PLR for video flows

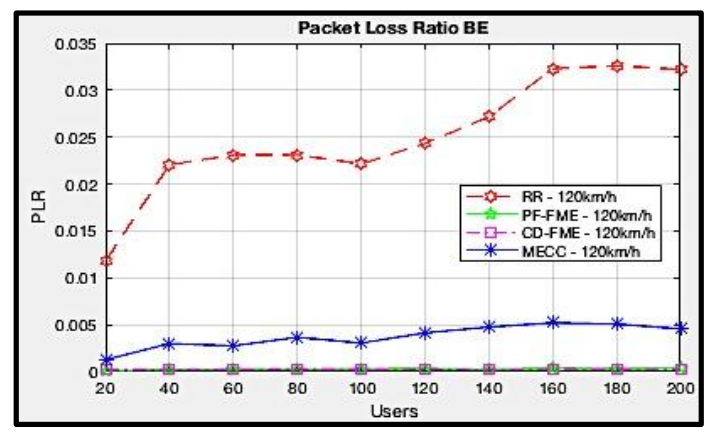

Figure 11. PLR for BE flows

\subsection{Fairness index}

The Jain fairness index of VoIP, video and, BE flows is illustrated in Figure 12, Figure 13 and Figure 14 respectively. The fairness value of CD-FME and PF-FME decreases as the number of users increases for all traffic flows. It is noticed that the fairness index for PF-FME is the highest among others between 60 to 80 users because the algorithm allocates resources to the user based on the ratio of the channel condition. Therefore, the users are being neglected when experiencing a bad channel condition. However, the fairness value for CD-FME decreased as the number of the user increases because resources are given only to users with the best channel condition and those with low channel quality will suffer from the starvation problem. The fairness value of MECC and RR is comparable to each other for all traffic flows and the value is kept between 0.7 and 0.8 as the number of the user increases. The fairness index for MECC and RR is steady, which is hovering between 0.7 and 0.8 . This is because RR and MECC schedulers are fair in terms of $\mathrm{RB}$ allocation.

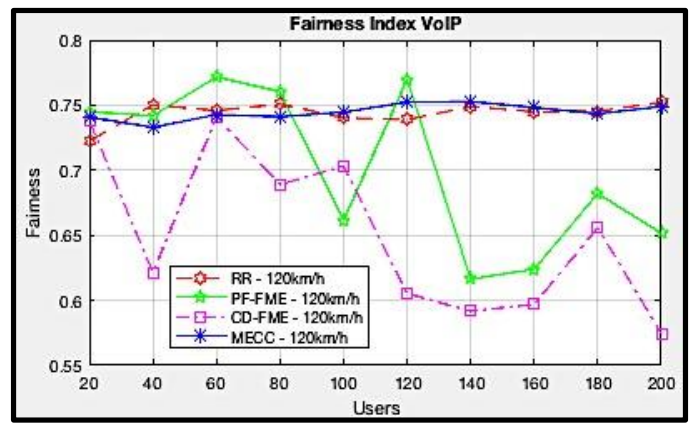

Figure 12. Fairness index for VoIP flows 


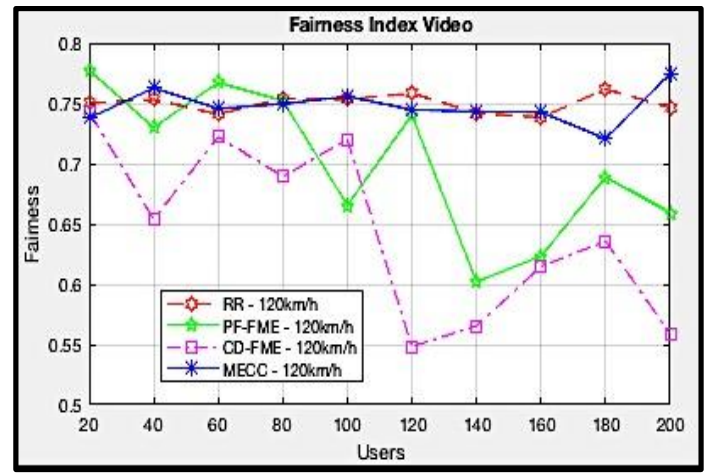

Figure 13. Fairness index for Video flows

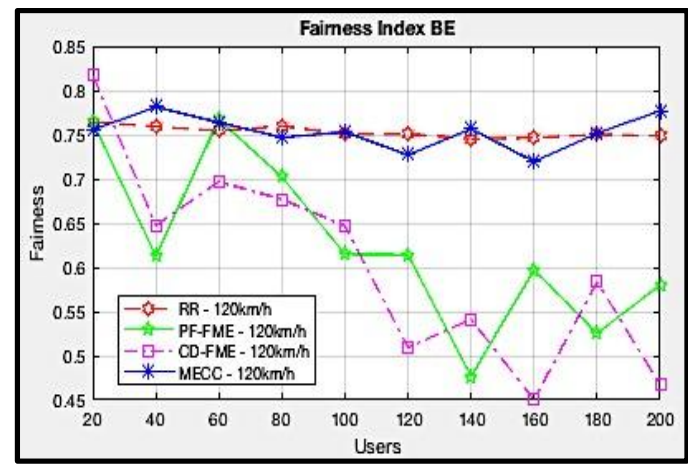

Figure 14. Fairness index for BE flows

\section{CONCLUSION}

This research has studied the performance of uplink scheduling algorithms for RT and NRT services using the LTE-SIM. The performance of MECC is compared to the CD-FME, PF-FME, and RR in terms of throughput, delay, packet loss ratio (PLR) and fairness index. The VoIP, video and BE flows is considered and the speed of the UEs is set to $120 \mathrm{~km} / \mathrm{h}$, which resembles the high-speed vehicular. From the simulations, it can be concluded that MECC algorithm is the best among the other algorithms as it delivers the highest throughput for all flows due to a fair allocation of RBs among the users. Thus, this contributes to low delay, low packet loss rate and delivers a satisfactory level of fairness value in the vehicular scenarios. Furthermore, it is proved that MECC is able to support 200 users without compromising the QoS parameters for the traffic flows of VoIP, video, and BE. Hence it can be concluded that MECC is the best scheduler in provisioning the QoS requirements for the RT and NRT traffics. As a future recommendation, the performance evaluation of the MECC algorithm will be studied in the static and pedestrian environment and compared to the other uplink-scheduling algorithm available in the literature.

\section{ACKNOWLEDGEMENTS}

We are grateful to the University Technology Mara (UiTM) Shah Alam, Selangor for the research grants of Bistari 600-IRMI/DANA 5/3/BESTARI (/2018) during the course of this research.

\section{REFERENCES}

[1] Angri I, Mahfoudi M, Najid A, Bekkali M El, "Exponential MLWDF ( EXP-MLWDF ) Downlink Scheduling Algorithm Evaluated in LTE for High Mobility and Dense Area Scenario," Int J Electr Comput Eng, 8(3):1618-28, 2018.

[2] Yadav SP, Bera SC., "PAPR Analysis of Single Carrier FDMA System for Uplink Wireless Transmission," 2015 10th Int Conf Information, Commun Signal Process ICICS 2015, 2016.

[3] Abu-Ali N, Taha AEM, Salah M, Hassanein H. "Uplink scheduling in LTE and LTE-advanced: Tutorial, Survey and Evaluation Framework," IEEE Commun Surv Tutorials, 16(3):1239-65, 2014.

[4] Farzaneh Y, Mardi A, Ghorashi SA., "A QoS-Aware Downlink Packet Scheduler Using Token Bucket Algorithm for LTE Systems," 22nd Iran Conf Electr Eng ICEE 2014, (Icee):1775-80, 2014.

[5] Piro G, Grieco LA, Boggia G, Capozzi F, Camarda P., "Simulating LTE Cellular Systems: an Open Source Framework," IEEE Trans Veh Technol. 1-16, 2010.

[6] Liu Z, Shen Y, Yu Z, Qin F, Chen Q., "Adaptive Resource Allocation Algorithm in Wireless Access Network. TELKOMNIKA (Telecommunication Comput Electron Control" [Internet]. 14(3):887, 2016. Available from: http://www.journal.uad.ac.id/index.php/TELKOMNIKA/article/view/3615

[7] Universal E, Co MA. Etsi Ts 1136 213. Etsi. 2015;V12.7.0.

[8] Ismail SB, Bt D, Ali M, Ya N., "Performance Analysis of Uplink Scheduling Algorithms in LTE Networks," Indones J Electr Eng Comput Sci, 9(2):373-9, 2018.

[9] De Temiño LAMR, Berardinelli G, Frattasi S, Mogensen P., "Channel-aware Scheduling Algorithms for SC-FDMA in LTE Uplink," IEEE Int Symp Pers Indoor Mob Radio Commun PIMRC. 2008.

[10] Elgazzar K, Salah M, Taha A-EM, Hassanein H., "Comparing uplink schedulers for LTE. Proc 6th Int Wirel Commun Mob Comput Conf ZZZ-IWCMC '10" [Internet]. 189, 2010.

Available from: http://portal.acm.org/citation.cfm?doid=1815396.1815441

[11] Safa H, Tohme K., "LTE Uplink Scheduling Algorithms: Performance and Challenges," 2012 19th Int Conf Telecommun ICT 2012, (Ict), 2012.

[12] Ahmed RE, Almuhallabi HM., "Throughput-fairness Tradeoff in LTE Uplink Scheduling Algorithms," 2016 Int 
Conf Ind Informatics Comput Syst CIICS 2016, 2016.

[13] Dardouri S, Bouallegue R., "Comparative Study of Scheduling Algorithms for LTE Networks," World Acad Sci Eng Technol Int J Comput Inf Sci Eng. 8(3):425, 2014. Available from: http://dx.doi.org/10.1007/s11277-0152289-2

[14] Janevski T, Member S, Jakimoski K., "Comparative Analysis of Packet Scheduling Schemes for HSDPA Cellular Networks," 100-3, 2008.

[15] Mahesha SN, Gopinath CY, R HKA, Kumar EN., "Performance Study of LTE Scheduling Algorithms in Vehicular and Pedestrian," 2016;3871-7.

[16] Noh KSSKM, Ali DM, Rahman AAA, Samingan AK, Yusuf Y, Yusuff NM, "Performance Analysis of EXP-BET Algorithm," 9(1):15-9.

[17] Chuah CN, Katz RH., "Characterizing Packet Audio Streams from Internet Multimedia Applications," IEEE Int Conf Commun, 2:1199-203, 2002.

[18] U.N.Nwawelu, C.I.Ani, M.A.Ahaneku, "Comparative Analysis of the Performance of Resource Allocation Algorithms in Long Term Evolution Networks," Niger J Technol, 36(1):163-71, 2017.

[19] Wheeb AH., "Performance Evaluation of UDP, DCCP, SCTP and TFRC for Different Traffic Flow in Wired Networks," Int J Electr Comput Eng. 7(6):3552-7, 2017.

[20] Specification T., TS 123203 - V9.3.0 - Digital cellular telecommunications system (Phase 2+); Universal Mobile Telecommunications System (UMTS); LTE; Policy and charging control architecture (3GPP TS 23.203 version 9.3.0 Release 9). 0:0-125, 2010.

[21] Yahiya TA., "Understanding LTE and its Performance," Springer New York; 1-277 p, 2011.

\section{BIOGRAPHIES OF AUTHORS}
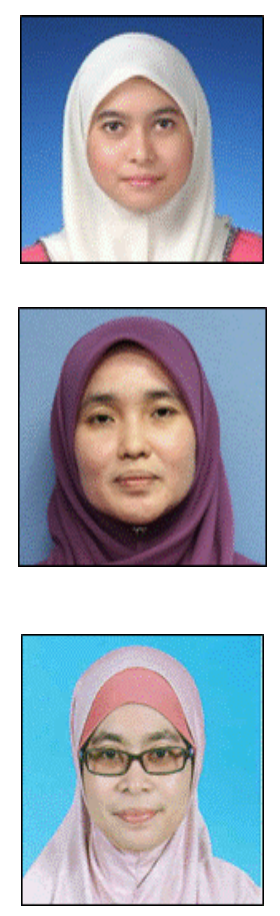

Shafinaz Ismail is a Ph.D. Candidate at the University Technology MARA (UiTM), Selangor, Malaysia. She has received the Master of Science in Telecommunication and Information Engineering in 2014 from University Technology MARA (UiTM), Selangor, Malaysia. She obtained her BEng degree from University Tun Hussein Onn Malaysia (UTHM) with Honours, in Electrical Engineering in 2009. Previously, she obtained her diploma from Polytechnic Sultan Abdul Halim Mu'adzam Shah (POLIMAS), Malaysia in Mechatronic Engineering, graduating in 2006.

Darmawaty Mohd Ali is a Senior Lecturer at the University Technology MARA (UiTM), Selangor, Malaysia. She obtained her Ph.D in 2012 from University Malaya, Malaysia. She has received the Master of Engineering in Electrical in 2002 from University Technology Malaysia. Previously, she obtained her first degree from University Kebangsaan Malaysia with Honours, in Electrical, Electronic and System, graduating in 1999. She is a member of Wireless Communication Technology (WiCOT) Research Interest Group (RIG) and her research interests include Wireless Access Technology and Quality of Service in Wireless Broadband.

Azita Laily Yusof is an Associate Professor in the Department of Communication Engineering, Universiti Teknologi MARA (UiTM). In 2011, she was awarded a Ph.D degree in Electrical, Electronic \& Systems Engineering from Universiti Kebangsaan Malaysia (UKM). She also obtained her M. Eng. degree from Universiti Kebangsaan Malaysia in Communications \& Computer Engineering in 2000. Previously, she obtained her first degree from Universiti Kebangsaan Malaysia with honours in Electrical, Electronic \& Systems Engineering in 1999. She has been appointed as the Programme Coordinator of FKE from 2013 until 2017. She has been awarded 17 research grants funded by the government and university. She has published over 70 journal papers and conference proceedings on various topics related to wireless communications technologies. She is a group member of Wireless Communication Technology (WiCoT) group at UiTM and a member of IEEE Communications Society. Her current research interests include radio resource and interference management in mobile communications networks. 